\title{
Template Synthesis of Hollow Carbon Nanofibers
}

\author{
Yian Song ${ }^{1}$, Jiake Wei ${ }^{1,2}$ and Jingyue Liu ${ }^{1}$ \\ 1. Department of Physics, Arizona State University, Tempe, AZ, 85287, USA \\ 2. The Institute of Physics, Chinese Academy of Sciences, Beijing, 100190, China
}

Hollow nanostructures, especially hollow carbon nanostructures with adjustable inner space and large surface area, have attracted increasing interest because of their potential applications in catalysis, energy storage, and drug delivery [1]. To better control the morphology of hollow nanostructures, template based synthesis approach is of interest [1]. ZnO nanostructures can serve as appropriate templates since various morphologies can be synthesized [2]. Moreover, the $\mathrm{ZnO}$ template can be removed by using reductive gas treatment at moderate temperatures. Here we report the synthesis of hollow carbon nanofibers (HCNFs) from $\mathrm{ZnO}$ nanowires (NWs) by using ethanol as the carbon source.

The key synthesis steps are schematically illustrated in Figure 1. First, the ZnO NWs were produced by a modified physical vapor deposition process. Then the as-synthesized $\mathrm{ZnO}$ NWs reacted with an ethanol vapor at a temperature between $500{ }^{\circ} \mathrm{C}$ to $700{ }^{\circ} \mathrm{C}$ to form a layer of carbonaceous material conformably covering the $\mathrm{ZnO}$ NWs. The thickness of the deposited layer can be adjusted by controlling the deposition time and temperature. The coated $\mathrm{ZnO}$ NWs were then reduced by $5 \% \mathrm{H}_{2}$ (with $\mathrm{N}_{2}$ or $\mathrm{Ar}$ as the carrier gas) at $850{ }^{\circ} \mathrm{C}$ for 3 hours to completely remove the $\mathrm{ZnO}$. The final high temperature treatment further graphitized the HCNFs and increased their total surface area.

Figure 2 shows SEM images of the as-prepared ZnO NWs (a) and the final HCNFs after the high temperature treatment (b). Figure $2 \mathrm{c}$ shows an annular dark-field image of the HCFs confirming that the produced carbon fibers are hollow. By analyzing many similar images of the different regions of the sample it was estimated that the average wall thickness of the HCFs is approximately 4-5 nm. Figure 2d, a high resolution bright-field STEM image, clearly reveals short segments of wavy lattice fringes, similar to those of graphite. The total surface area of the synthesized HCFs was measured to be approximately $1970 \mathrm{~m}^{2} / \mathrm{g}$, far exceeding the original total surface area $\left(\sim 12-18 \mathrm{~m}^{2} / \mathrm{g}\right)$ of the $\mathrm{ZnO} \mathrm{NWs}$.

Electron energy loss spectroscopy (EELS) technique was used to probe the electronic structure of the synthesized HCFs. Figure 2e shows the EELS spectra of the carbon K-edge acquired from a HCNF after high temperature treatment, amorphous carbon support of the TEM grid, and graphite (taken from reference [3]). The first edge of the carbon K-edge spectra corresponds to the $1 \mathrm{~s}$ to $\pi^{*}$ transition and the $2^{\text {nd }}$ edge corresponds to $1 \mathrm{~s}$ to $\sigma^{*}$ transition. The relative peak intensities of these two peaks can be used to quantitatively measure the $\mathrm{sp}^{3} / \mathrm{sp}^{2}$ ratio of the synthesized HCNFs. Figure 2e suggests that after the high temperature treatment the HCNFs were highly graphitized with an electronic structure close to that of crystalline graphite. Spatial resolved EELS analysis of the HCNFs after different reduction or chemical treatments can provide useful information on the evolution of the electronic properties of the HCNFs. The approach reported here can be used to produce various types of carbon hollow nanostructures for practical applications, for example, lithium based battery or carbon based nanocatalysts.

References:

[1] X Lou, et al, Advanced Materials 20 (2008), p. 3987.

[2] Z Wang, Journal of Physics: Condensed Matter 16 (2004), p. R829. 
[3] K. Andre Mkhoyan, et al, Nano Letters, 9 (2009), p.1058

[4] The authors acknowledge funding by the College of the Liberal Arts and Science of Arizona State University and the use of facilities in the John M. Cowley Center for High Resolution Electron Microscopy at Arizona State University. The help from Mr. Daniel Mieritz and Professor Don Soe for suface area measurement is appreciated.

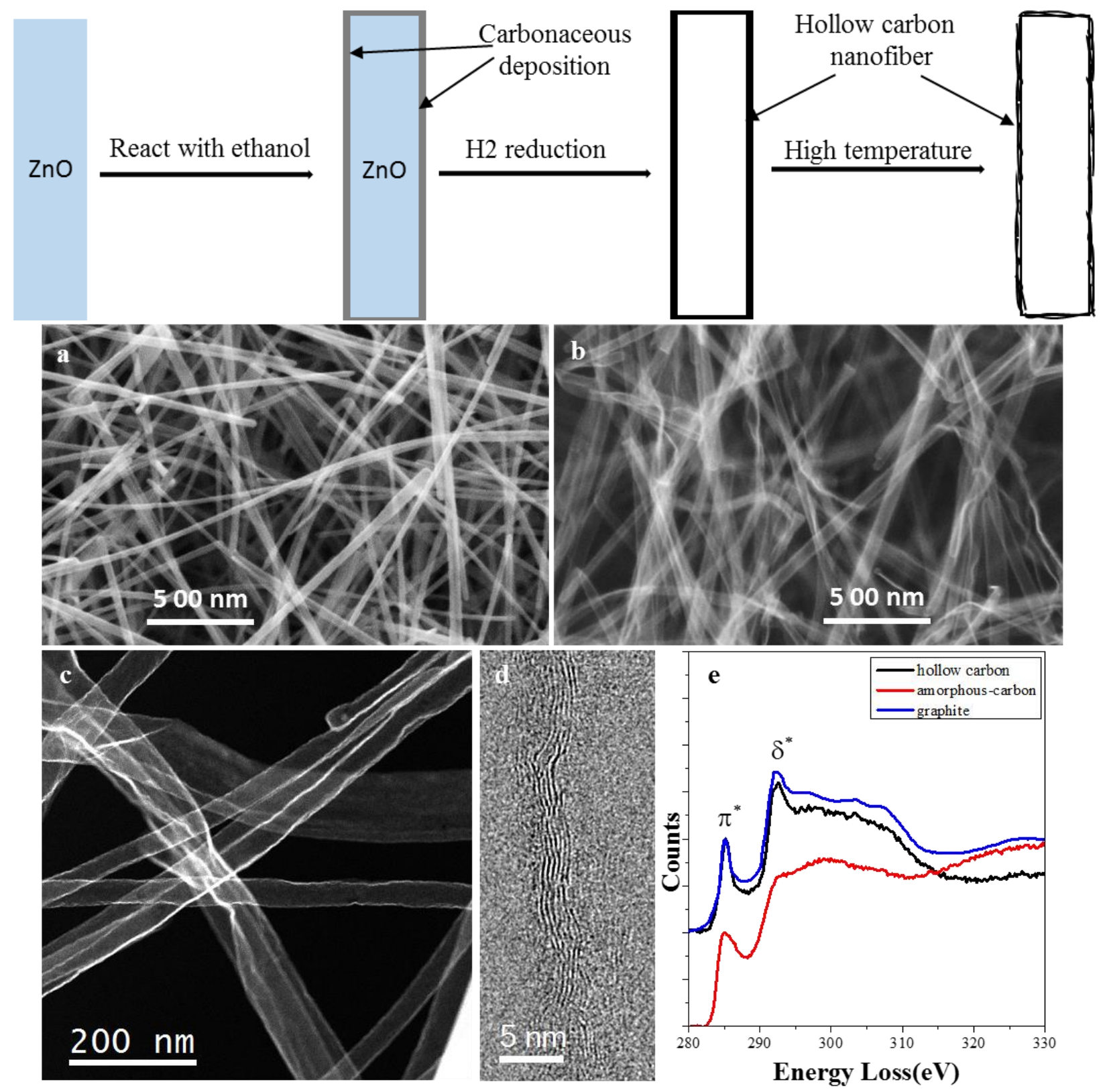

Figure 1. Illustration of the synthesis process for producing carbon hollow nanofiber.

Figure 2. SEM images of (a) ZnO NWs and (b) HCNFs after high temperature treatment; (c) ADF image of the HCFs showing the thickness of the HCNF walls; (d) bright-field STEM image of the wall of the HCF showing the segmented graphite-like fringes; (e) carbon K-edge EELS spectrum of a HCF together with amorphous carbon and graphite. The spectra in (e) were normalized by the intensity of the carbon $\pi^{*}$ peak, and both the HCF and the reference graphite peaks were shifted upward for clarity. 